\section{Homöopathie steht hoch im Kurs}

In einer Online-Umfrage gab die Mehrheit der 2000 Teilnehmer an, dass sie Gebrauch von homöopathischen Arzneimitteln macht. Mehr als 50\% waren darüber hinaus „zufrieden“ bis „sehr zufrieden“ mit der Wirksamkeit. Pressemitteilung Dtsch Arztebl, 28.02.11

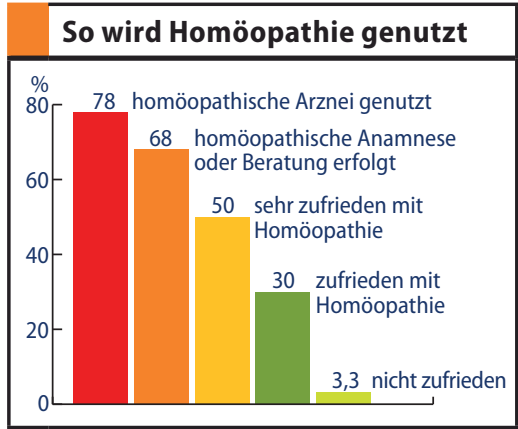

\section{Mehr Schlaganfälle durch Diät-Getränke}

Diät-Limos schmecken gut und sind kalorienarm, steigern aber das Risiko für kardiovaskuläre Ereignisse, so die Auswertung einer amerikanischen Studie. Um 48\% erhöhte der häufige Konsum die Gefahr, einen Myokardinfarkt oder Schlaganfall zur erleiden - und das nach Ausschluss von Faktoren wie metabolisches Syndrom oder periphere Gefäßerkrankung. Welche Inhaltsstoffe den Anstieg bewirken, ist allerdings noch unklar.

Gardener H et al, Stroke 2011 (online first)

\section{SpringerMedizin via Twitter folgen}

Obwohl der Frühling noch nicht Einzug gehalten hat, wird das Zwitschern immer lauter: Werden Sie zum „Follower" und "lauschen" Sie uns bei Twitter. twitter.com/springermedizin
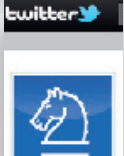

\section{Runter mit der Temperatur! Kühle Räume fördern das Abspecken}

Eine Vorliebe für Schokotorte, Pommes und Cola, stundenlanges Sitzen auf Bürostuhl und Fernsehcouch - das sind sicher die Hauptgründe für die grassierende Adipositas-Epidemie. Doch auch (zu) gut geheizte Räume könnten Übergewicht begünstigen.
Kann man dem Hüft- und Bauchspeck zu Leibe rücken, indem man die Heizung herunterdreht? Das stellen britische Forscher zur Diskussion: Demnach gehört das Wohnen in überwärmten Räumen zu den Ursachen, warum viele Menschen zu dick sind. In den vergangenen Jahrzehnten sei die Raumtemperatur in den USA und anderen hoch entwickelten Ländern stetig gestiegen, berichten die Wissenschaftler vom University College London.

Mit dem Einbau von Zentralheizungen in immer mehr Häuser gleichen sich für die Bewohner die Temperaturen in Sommer und Winter zunehmend anei- nander an. Folglich gewöhnen sie sich an die Wärmegrade, sodass sie bei einer Abkühlung früher zu frösteln beginnen.

\section{Warme Råume doppelt schlecht}

Wer aber tiefe Temperaturen scheut, könnte auf zweierlei Art und Weise Fettpolster begünstigen: Zum einen wird der Umsatz von Energie, die zum Warmhalten des Körpers benötigt würde, heruntergeschraubt. Zum anderen verliert der Organismus nach und nach grundsätzlich die Fähigkeit, selbst Wärme zu produzieren.

Johnson F et al, Obes Rev 2011 (online first)

\section{Nierenverkalkung und Harnflut drohen Experten warnen: Vitamin D nur für Risiko-Gruppen!}

\begin{abstract}
Endokrinologen warnen vor Überbewertung eines Vitamin-D-Mangels. Die Einnahme künstlicher VitaminPräparate sei daher nur in seltenen Fällen indiziert. Statt dessen geben die Ärzte folgenden Rat: Ab ins Freie - auch im Winter!
\end{abstract}

„Gefährliche Mangelerscheinungen sind in Deutschland erfreulicherweise selten" erklärte Prof. Helmut Schatz von der Deutschen Gesellschaft für Endokrinologie (DGE). Vitamin-Präparaten solle man deshalb mit Vorsicht begegnen. Nur sogenannte Risikogruppen wie
z.B. ältere Menschen mit OsteoporoseNeigung oder Säuglinge hätten einen echten Nutzen von der Substitution.

\section{Tageslicht ist angesagt}

Für Normalpersonen berge die zusätzliche Vitamin-D-Einnahme sogar eher Gesundheitsrisiken, so der Experte. Hohe Dosen führen zu einer Steigerung des Kalzium-Spiegels im Blut mit Harnflut, Nierenverkalkung, Übelkeit und Erbrechen. Viel besser sei dagegen der tägliche Aufenthalt im Freien. (cd) 